\title{
Dynamic Simulation of Mixing-limited Symmetric and Asymmetric Patterns in Homogeneous Autocatalytic Reactors
}

\author{
Anwesha Chaudhury, Saikat Chakraborty*
}

\section{Summary}

In this paper we study the dynamic evolution of mixing-limited patterns in homogeneous autocatalytic reactors. Using a regularized low dimensional model, our previous works $[1,2,3]$ mainly focused on the symmetric patterns that develop due to the difference in mixing and reaction time scales, while our present paper simulates the formation of asymmetric patterns, which have been observed in experimental studies [4]. We find that higher eigen modes of initial perturbation lead to patterns that are more stable and more asymmetric than those formed from lower modes.

Keywords

Multiscale analysis, Dynamics and control of chemical reacting systems, Complex reacting flows.

\section{Introduction}

Transport-limited patterns are formed in autocatalytic reaction systems when a spatially uniform steady state loses its stability to transverse perturbations, and the patterns, once formed, are sustained when local mixing is much slower than reaction. The formation of patterned states may often reduce the product yield by deactivation of the catalyst or reactor runaway. Using a regularized low dimensional model, our previous works $[1,2,3]$ focused on the symmetric patterns that develop due to the difference in mixing and reaction time scales, while our present paper simulates the formation of asymmetric patterns, typically observed in experimental studies [4].

\section{Mathematical Modeling}

We consider an isothermal autocatalytic reaction of the type $\mathrm{A}+2 \mathrm{~B} \rightarrow 3 \mathrm{~B}$ occurring in a tubular reactor of radius $a$ and length $L$. Starting from the unsteady state 3-D convection-diffusion- reaction (CDR) equation, we obtain a 2-D low dimensional model using Liapunov-Schmidt based spatial-averaging technique [5], followed by regularization [6]. The resultant axially-averaged model is given in terms of the axially-averaged reactant concentration $c_{i}$ as

$p\left[\frac{\partial \bar{c}_{i}}{\partial t}+\left(\bar{c}_{i}-c_{i, i n}\right)\left(1+\frac{D a}{6 \beta}\right)+\operatorname{Da} \cdot r\left(\overline{c_{i}}\right)\right]=\frac{1}{\xi} \frac{\partial}{\partial \xi}\left(\xi \frac{\partial \bar{c}_{i}}{\partial \xi}\right)+\frac{1}{\xi^{2}} \frac{\partial^{2} \bar{c}_{i}}{\partial \theta^{2}}$,

where $i=A, B, \mu_{A}=1, \mu_{B}=C_{B, i n} / C_{A, i n \text {, and }}$

$c=\frac{C}{C_{R}}, \xi=\frac{\xi}{a}, z=\frac{x}{L}, t=\frac{t}{\tau_{C}}, r(c)=\frac{R(C)}{R\left(C_{R}\right)}, \mu_{i}=\frac{C_{i, i n}}{C_{A, i n}}, p=\frac{t_{D}}{\tau_{C}}=\frac{a^{2} u_{x}^{\prime}}{L D_{m}}$,

$P e=\frac{t_{Z}}{\tau_{C}}=\frac{u_{x}^{\prime} L}{D_{m}}, D a=\frac{\tau_{C}}{t_{R}}=\frac{L R\left(C_{R}\right)}{u_{x}^{\prime} C_{R}}, \beta=\frac{D a}{P e}=\frac{D_{m} R\left(C_{R}\right)}{u_{x}^{\prime 2} C_{R}}=\frac{\tau_{c}^{2}}{t_{R} t_{Z}}$.

The boundary and initial conditions are given by

$\left.\bar{c}\right|_{\xi=0}$ is finite, $\left.\frac{\partial c}{\partial \xi}\right|_{\xi=1}=0, \overline{c_{i}}(\xi, \theta, t)=\bar{c}_{i}(\xi, \theta+2 \pi, t)$,

$c_{i}(\xi, \theta, t=0)=c_{i 0}(\xi, \theta)$.

\section{Steady State Results}

Using the regularized model, the steady state concentration of $B\left(\bar{c}_{B, s s}\right)$ is obtained by solving
$\left[\frac{D a}{1+D a / 6 \beta}\right] \bar{c}_{B, s s}^{3}-\left[\frac{D a(1+\mu)}{1+D a / 6 \beta}\right] \bar{c}_{B, s s}^{2}+\bar{c}_{B, s s}-\mu=0$,

and the steady state concentration of $A\left(\bar{c}_{A, s S}\right)$ is given by

$\bar{c}_{A, s S}=1+\mu-\bar{c}_{B, s s}$

For fixed values of $D a, \beta$ and $\mu$, the above relation can be used to determine $\bar{c}_{A, s s}$ and $\bar{c}_{B, s s}$. As shown in Fig. 2(b), the bifurcation diagrams (of conversion, $X_{A}$ vs Damköhler number, $D a$ ) obtained by solving eqns. (5) and (6) are $\mathrm{S}$ shaped in nature and suggest the existence of multiple (three) solutions, which is dependant on $\beta$ or $\mu$, and increases with increasing $\mu$ and decreasing $\beta$ (Fig. 2.b).

The hysteresis loci for the system are in terms of $D a, P e$ and $\mu$ as

$\bar{c}_{B, s s}=\frac{a(1+\mu) \pm \sqrt{a^{2}(1+\mu)^{2}-3 a}}{3 a}$,

where, $a=D a /(1+P \%)$. The region between the two curves in the hysteresis loci in Fig. 1 corresponds to the unstable middle branch as shown in the bifurcation diagram.

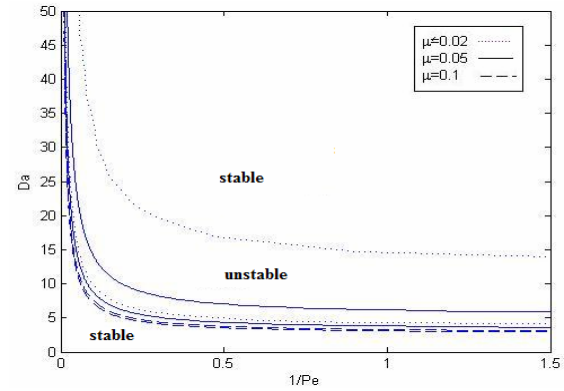

Fig. 1. Hysteresis loci for $\beta=20$ and different values of $\mu$.

The hysteresis loci along with the bifurcation diagrams suggest that higher the $\mu$, smaller is the region of instability (Fig. 2b).

A stability analysis is performed on the steady state solution and neutral stability plots are obtained for several

* Corresponding author. Email: dr.s.chakraborty@gmail.com, Phone: +91-32222-83930, Fax: +91-32222-82250 
values of $p, D a, P e$ and $k_{m n}$. For any integer value of $m$ and $n$, the neutral stability relation is obtained as

$\left(\frac{k_{m n}^{2}}{p}\right)^{2}+\left[2 \kappa+D a \cdot \bar{c}_{B, s s}\left(\bar{c}_{B, s s}-2 \bar{c}_{A, s s}\right)\right]\left(\frac{k_{m n}^{2}}{p}\right)+\left[\kappa^{2}+D a \cdot \bar{c}_{B, s s}\left(\bar{c}_{B, s s}-2 \bar{c}_{A, s s}\right) \kappa\right]=0$,

where, $\kappa=1+D a / 6 \beta$. The neutral stability curves have been plotted for different values of $\mu, \beta$ and $k_{m n}$ (Fig. 2a) and the observations from these cases reaffirms the conclusions drawn from the bifurcation or hysteresis diagrams. Besides, our neutral stability studies [1] also show that higher eigen modes are excited only from significant transverse mixing limitations (large $p$ ) in the reactor (Fig. 2a).

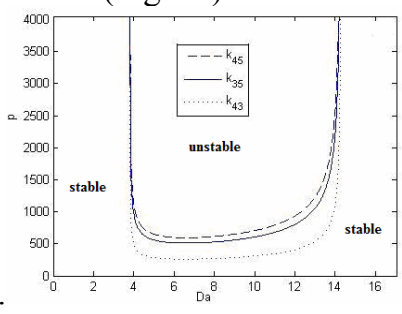

(a)

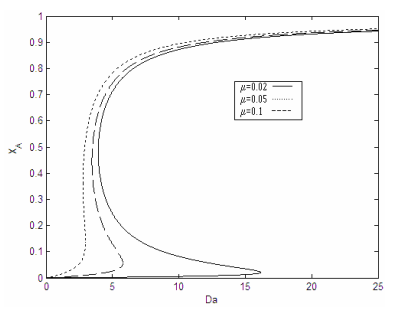

(b)
Fig. 2. (a) Neural stability diagram for higher eigen modes at $\mu=0.02$, (b) Bifurcation diagrams for $\beta=20$ and different values of $\mu$

\section{Dynamic Simulation of Patterns}

The dynamics of pattern formation have been studied by simulating the regularized low dimensional model and analyzing its behavior in response to small perturbation of less than $7 \%$ of the steady state concentration. This leads us to the observation that symmetric and asymmetric patterns are formed for lower and higher eigen modes respectively. Higher the eigen mode, more stable is the pattern. Also, asymmetry is observed for patterns pertaining to eigen modes higher than $m=2, n=2$. It may be noted that the patterns emerging due to the initial perturbation are quite different from the stable patterns that evolve eventually. Fig. 4 shows the stable patterns that emerge with time. The time required to attain stable patterns varies from $5 \tau$ to $15 \tau$, where $\tau$ is the reactor residence time.

The asymmetric patterns we obtain can be broadly classified into the following categories:

1. Target patterns evolving to band patterns corresponding to mode numbers $\mathrm{k}_{04}, \mathrm{k}_{12}, \mathrm{k}_{22}, \mathrm{k}_{32}$.

2. Band patterns changing direction corresponding to mode numbers $\mathrm{k}_{34}, \mathrm{k}_{43}, \mathrm{k}_{52}, \mathrm{k}_{53}$, $\mathrm{k}_{54}, \mathrm{k}_{55}$.

3. Antiphase patterns evolving to band patterns corresponding to mode numbers $\mathrm{k}_{35}, \mathrm{k}_{42}$.

4. Asymmetric antiphase patterns corresponding to mode numbers $\mathrm{k}_{24}, \mathrm{k}_{25}$, $\mathrm{k}_{33}$.

Our simulations show that the symmetric and asymmetric patterns emerge from the unstable middle branch of the Sshaped bifurcation curves in response to small perturbations to the uniform steady states. The patterns undergo a process of concentration segregation before attaining stability. The symmetric patterns are typically of the form band, antiphase or target whereas the asymmetric patterns are formed by the superposition of two or more of these simple symmetric forms.

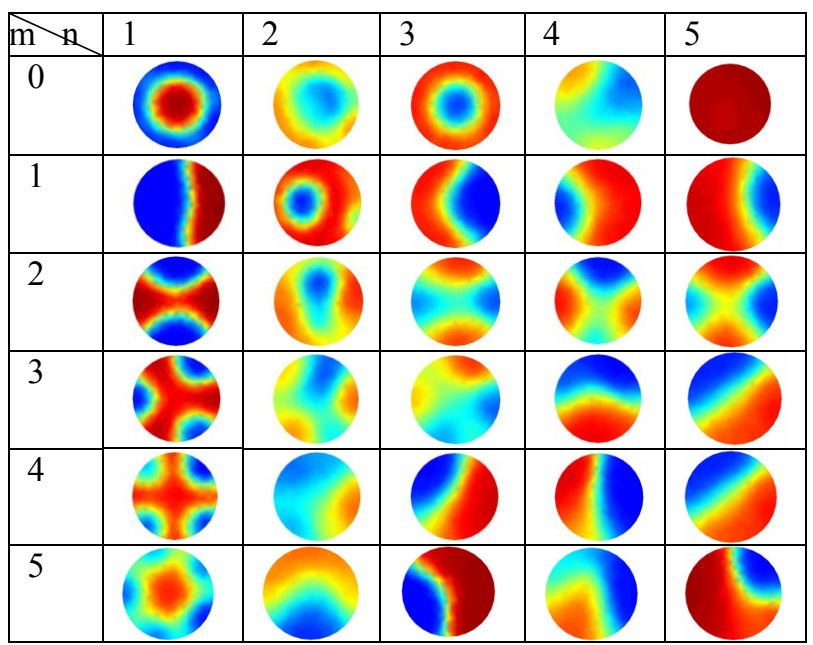

Fig. 4. Stable patterns for various $\mathrm{k}_{\mathrm{mn}}$ values

However, the patterns formed from perturbations of higher eigen modes are significantly more stable and asymmetric than those formed from lower eigen modes, and are remarkably different from the initial perturbed states. We have performed a detailed parametric analysis of the patterns to quantify the effects of the parameters $P e, p, D a$ on the evolution of patterns and its stability. We find that increased transverse or axial mixing limitations (quantified by increasing $p$ and $P e$, respectively) result in patterns that are formed sooner and attain stability faster.

\section{References}

(1) Gupta, A; Chakraborty, S. Linear stability analysis of high- and low-dimensional models for describing mixing limited pattern formation in homogeneous autocatalytic reactors. Chem. Engg. J. 2009, 399-411.

(2) Gupta, A; Chakraborty, S. Dynamic simulation of mixing-limited pattern formation in homogeneous autocatalytic reactions. Chemical Product and Process Engg. 2008, 3(2), Article 9.

(3) Gupta, A; Chakraborty, S. Temporal evolution of mixing-limited spatial patterns in nonisothermal homogeneous reactor. J. Chem. Engg. Japan. 2009, ISCRE 20 Special edition. in press.

(4) Marwaha, B; Luss, D. Formation and dynamics of a hot zone in radial flow reactor. AIChE J 2002, 617-624.

(5) Chakraborty, S; Balakotaiah, V. Low-dimensional models for describing mixing effects in laminar flow tubular reactors. Chem. Engg. Sci. 2002, 2545.

(6) Chakraborty, S; Balakotaiah, V. Spatially averaged Multiscale Models for Chemical Reactors, Advances in Chemical Engineering 2005, Vol. 30 